\title{
DATA-DRIVEN FAILURE PREDICTION IN BRITTLE MATERIALS: A PHASE FIELD-BASED MACHINE LEARNING FRAMEWORK
}

\author{
Eduardo A. Barros de Moraes, ${ }^{1,2}$ Hadi Salehi, ${ }^{1} \mathcal{E}$ \\ Mohsen Zayernouri ${ }^{1,3, *}$
}

${ }^{1}$ Department of Mechanical Engineering, Michigan State University, $428 \mathrm{~S}$

Shaw Ln, East Lansing, MI 48824, USA

${ }^{2}$ Department of Computational Mathematics, Science, and Engineering, Michigan State University, 428 S Shaw Ln, East Lansing, MI 48824, USA

${ }^{3}$ Department of Statistics and Probability, Michigan State University, East Lansing, MI 48824, USA

*Address all correspondence to: Mohsen Zayernouri, Department of Mechanical Engineering, Michigan State University, 428 S Shaw Ln, East Lansing, MI 48824, USA; Tel.: +1 517353 0825, E-mail: zayern@msu.edu

Original Manuscript Submitted: 3/2/2020; Final Draft Received: 6/30/2020

Failure in brittle materials led by the evolution of micro- to macro-cracks under repetitive or increasing loads is often catastrophic with no significant plasticity to advert the onset of fracture. Early failure detection with respective location are utterly important features in any practical application, both of which can be effectively addressed using artificial intelligence. In this paper, we develop a supervised machine learning $(M L)$ framework to predict failure in an isothermal, linear elastic and isotropic phase-field model for damage and fatigue of brittle materials. Time-series data of the phase-field model is extracted from virtual sensing nodes at different locations of the geometry. A pattern recognition scheme is introduced to represent time-series data/sensor node responses as a pattern with a corresponding label, integrated with $M L$ algorithms, used for damage classification with identified patterns. We perform an uncertainty analysis by superposing random noise to the time-series data to assess the robustness of the framework with noise-polluted data. Results indicate that the proposed framework is capable of predicting failure with acceptable accuracy even in the presence of high noise levels. The findings demonstrate satisfactory performance of the supervised $M L$ framework and the applicability of artificial intelligence and $M L$ to a practical engineering problem, i.e., data-driven failure prediction in brittle materials.

KEY WORDS: finite element method, virtual sensing nodes, pattern recognition, artificial neural networks, $k$-nearest neighbor, confusion matrix, failure location/pattern

\section{INTRODUCTION}

Predictability is essential to any mathematical model for failure and fracture. From early linear elastic fracture mechanic models from Griffith (1921) to failure analysis through damage 
mechanics by Lemaitre and Desmorat (2005), numerical models have improved in scope and complexity to provide realistic simulations of material failure to meet industry goals of safety and to reduce component weight and production costs. The accurate simulation of the failure process, from crack initiation to propagation until final failure, in a consistent way, while respecting the physics and developing robust numerical methods, is still a challenging task.

During the last decade, phase-field models have been successfully established as a powerful tool in the study of damage and fatigue. By modeling sharp interfaces through smooth continuous fields, the dynamics of moving boundaries using phase fields emerged in diverse physical applications, including fluid separation (Cahn and Hilliard, 1958), solidification (Allen and Cahn, 1979), tumor growth (Lima et al., 2014), two-phase complex fluid flow (Yue et al., 2004), and fluid-structure interaction (Sun et al., 2014). In failure analysis, crack sharpness is modeled through a smooth phase field, indicating the state of the material among fractured, virgin, and intermediate damaged zones, evolving through Allen-Cahn-type equations. Examples ranging from brittle (Borden et al., 2014; Miehe et al., 2010a,b), ductile (Ambati et al., 2015, 2016), and dynamic fracture (Borden et al., 2012; Hofacker and Miehe, 2013) successfully described phenomenological effects such as crack initiation, branching, and coalescence. The inclusion of fatigue effects was initially attempted with Ginsburg-Landau free-energy potentials (Amendola et al., 2016) and fractional derivatives (Caputo and Fabrizio, 2015). A more general framework for damage and fatigue was later developed in a nonisothermal and thermodynamically consistent approach (Boldrini et al., 2016; Chiarelli et al., 2017; Haveroth et al., 2018), followed by the emergence of further phase-field models for fatigue (Carrara et al., 2019; Seiler et al., 2019). Within this myriad of different models, solution uncertainty and parametric sensitivity are still influential, and the predictability of phase-field models for arbitrary conditions is yet a withstanding effort (Barros de Moraes et al., 2020). One promising approach to address the predictability of numerical models is to use artificial intelligence (AI), which has been consistently expanding its applicability over the years.

$\mathrm{AI}$ and machine learning (ML) have been widely used in different engineering applications such as structural health monitoring (Al Azzawi et al., 2016; Liu et al., 2011; Saeidpour et al., 2018; Salehi and Burgueno, 2018; Salehi et al., 2018a,b, 2019a,b; Santos et al., 2016; Silva et al., 2016; Wootton et al., 2017) and fatigue crack detection (Lim and Sohn, 2018; Lim et al., 2018; Rovinelli et al., 2018). ML algorithms, in the context of failure analysis, have been used for numerous applications, including phase-field models of polymer-based dielectrics (Shen et al., 2019), phase-field models of solidification (Yabansu et al., 2017), and crystal plasticity (Papanikolaou et al., 2019). Another interesting application of ML is to obtain a data-driven representation of free-energy potentials in the atomic scale and upscale it to a phase-field model using integrable deep neural networks (Teichert et al., 2019). Specifically for brittle failure, ML has been recently used to build surrogate models based on explicit crack representation (Hunter et al., 2019) and in failure prediction using a discrete crack representation model for high-fidelity simulations that feed an artificial neural networks (ANN) algorithm (Moore et al., 2018; Schwarzer et al., 2019). Nonetheless, the noted studies have only shown the applicability of ML in failure analysis. Therefore, ML methods have not yet been explored in the context of phase-field models for damage. It is noted that the use of ML leads to a new paradigm of phase-field modeling, where we establish the basis for novel data-driven frameworks, allowing systematic infusion of statistical information and corresponding uncertainty propagation from microscale models and experiments into continuum macroscopic failure models.

In this work, we develop an ML algorithmic framework for failure detection and classification merging a pattern recognition (PR) scheme and ML algorithms applied to a damage and 
fatigue phase-field model. We consider an isothermal linear elastic and isotropic material under the hypothesis of small deformations and brittle fracture. We simulate the phase field model using the finite element method (FEM) and a semi-implicit time-integration scheme to generate time-series data of damage phase-field $\varphi$ and degradation function $g(\varphi)=(1-\varphi)^{2}$ from virtual sensing nodes positioned at different locations across a test specimen. We introduce a PR scheme as part of the ML framework, in which time-series data from the FEM node responses are considered as a pattern with a corresponding label. We define multiple labels for "no failure," "onset of failure," and "failure" of the test specimen based on tensile test load-displacement curve and damage threshold concept. Once the patterns representing different states of the material are identified, the proposed ML framework employs $k$-nearest neighbor $(k-\mathrm{NN})$ and ANN algorithms to detect the presence and location of failure using such patterns. The use of ML algorithms makes the failure prediction framework practical even in cases of complex loading conditions, beyond the canonical example of monotonic loads presented here.

In this study, we consider different failure types to further assess the performance of the framework. In addition, by introducing noise to the time-series data, we ascertain the robustness of the proposed framework with noise-polluted data, leading to the effective use in failure analysis under high sensitive/uncertain parameters and operators. The idea of propagating uncertainty through the solution, combined with the inherent nonlinearity of the damage phase-field model make the choice of ANN particularly attractive in the construction of the framework. The findings from this study will pave a way for the development of novel data-driven failure prediction frameworks, which are able to efficiently establish a link among the classification results (i.e., accuracy) and different phase-field model parameters, thus enabling the computational framework to identify those parameters affecting the model's accuracy and updating them to achieve the best performance.

The paper is organized as follows: in Section 2 we present the damage and fatigue phasefield model, which is used to generate time-series data for the ML framework. We introduce the data generation procedure and corresponding label definitions in Section 3. We present the ML framework in Section 4, where we describe the integration of a pattern recognition scheme with the applied classification algorithms, $k$-NN and ANN. We present and discuss the numerical results in Section 5. We then conclude and summarize the paper in Section 6.

\section{DAMAGE AND FATIGUE PHASE-FIELD MODEL}

\subsection{Governing Equations}

We consider an isothermal phase-field framework for structural damage and fatigue, modeled by a system of coupled differential equations for the evolution of displacement $\mathbf{u}$, velocity $\mathbf{v}=\dot{\mathbf{u}}$, damage $\varphi$, and fatigue $\mathcal{F}$. The damage phase field $\varphi$ describes the volumetric fraction of degraded material and takes $\varphi=0$ for virgin material, $\varphi=1$ for fractured material, varying between those states, $0 \leq \varphi \leq 1$, as a damaged material. The evolution equation for the damage field is of the Allen-Cahn type, since the damage and aging effects are nonconservative and nondecreasing, and is derived along with the equations of motion for $\mathbf{u}$ and $\mathbf{v}$ through the principle of virtual power and entropy inequalities with thermodynamic consistency (Boldrini et al., 2016). The fatigue field $\mathcal{F}$ is associated to the presence of microcracks and is treated as an internal variable whose evolution equation is obtained through constitutive relations that must satisfy the entropy inequality for all admissible processes. The geometry is defined over a spatial domain $\Omega \subset \mathbb{R}^{d}, d=1,2,3$, at time $t \in(0, T]$.

Volume 2, Issue 1, 2021 
The final form of the governing equations will be defined by the choice of free-energy potentials related to elasticity, damage, and fatigue. We consider a linear elastic isotropic material, where the phase field free energy takes the usual gradient form:

$$
\Psi(\mathbf{E}, \varphi, \mathcal{F})=\frac{1}{2}(1-\varphi)^{2} \mathbf{E}^{T} \mathcal{C} \mathbf{E}+g_{c} \frac{\gamma}{2}|\nabla \varphi|^{2}+\mathcal{K}(\varphi, \mathcal{F})
$$

where $\mathbf{E}=\nabla^{S} \mathbf{u}$ is the strain tensor and $\nabla^{S} \mathbf{q}=\operatorname{sym}(\nabla \mathbf{q})$ represents the symmetric part of the gradient of a given vector field $\mathbf{q}$. Also, $\mathcal{C}$ is the elasticity tensor written in terms of the Young modulus $E$ and Poisson coefficient $\nu, g_{c}$ is the Griffith energy, $\gamma>0$ is the phase-field layer width parameter, and $\mathcal{K}(\varphi, \mathcal{F})$ is a function that models the damage evolution due to fatigue effects. The first term in Eq. (1) represents the degraded elastic response, modeled by the choice of degradation function $g(\varphi)=(1-\varphi)^{2}$. The final set of governing equations, defined over $\Omega \times(0, T]$, becomes

$$
\left\{\begin{array}{l}
\dot{\mathbf{u}}=\mathbf{v} \\
\dot{\mathbf{v}}=\operatorname{div}\left((1-\varphi)^{2} \frac{\mathcal{C}}{\rho} \mathbf{E}\right)+\frac{b}{\rho} \operatorname{div}(\mathbf{D})-\frac{\gamma g_{c}}{\rho} \operatorname{div}(\nabla \varphi \otimes \nabla \varphi)+\mathbf{f}, \\
\dot{\varphi}=\frac{\gamma g_{c}}{\lambda} \Delta \varphi+\frac{1}{\lambda}(1-\varphi) \mathbf{E}^{T} \mathcal{C} \mathbf{E}-\frac{1}{\lambda \gamma}\left[g_{c} \mathcal{H}^{\prime}(\varphi)+\mathcal{F} \mathcal{H}_{f}^{\prime}(\varphi)\right], \\
\dot{\mathcal{F}}=-\frac{\hat{F}}{\gamma} \mathcal{H}_{f}(\varphi),
\end{array}\right.
$$

subjected to appropriate initial and boundary conditions, which depend on the physical problem. Either displacement or stress are known at the boundaries, in addition to considering $\nabla \varphi \cdot \mathbf{n}=$ 0 on $\partial \Omega$. Moreover, the $\otimes$ operator denotes the outer product, the infinitesimal strain rate tensor is represented by $\mathbf{D}=\nabla^{S} \mathbf{v}$, and parameters $b$ and $\rho$ are the material's viscous damping and density, respectively. We construct $\lambda$ such that the rate of change of damage increases with damage (see, e.g., Lemaitre and Desmorat, 2005):

$$
\frac{1}{\lambda}=\frac{c}{(1+\delta-\varphi)^{\varsigma}},
$$

where $c, \varsigma>0$ are material dependent, and $\delta>0$ is a small constant to avoid numerical singularity.

The potentials $\mathcal{H}(\varphi)$ and $\mathcal{H}_{f}(\varphi)$ model the damage transition from 0 to 1 as fatigue changes from zero to $g_{c}$. We take their (ordinary) derivatives with respect to $\varphi$ to obtain potentials $\mathcal{H}^{\prime}(\varphi)$ and $\mathcal{H}_{f}^{\prime}(\varphi)$. Further details on fatigue potentials can be found in Boldrini et al. (2016). Choosing the transition to be continuous and monotonically increasing, suitable choices for the potentials are

$$
\begin{gathered}
\mathcal{H}(\varphi)= \begin{cases}0.5 \varphi^{2} & \text { for } 0 \leq \varphi \leq 1, \\
0.5+\delta(\varphi-1) & \text { for } \quad \varphi>1, \\
-\delta \varphi & \text { for } \varphi<0 .\end{cases} \\
\mathcal{H}_{f}(\varphi)=\left\{\begin{array}{lll}
-\varphi & \text { for } \quad 0 \leq \varphi \leq 1, \\
-1 & \text { for } \quad \varphi>1, \\
0 & \text { for } \quad \varphi<0 .
\end{array}\right.
\end{gathered}
$$


The evolution of fatigue $\mathcal{F}$ is controlled by $\hat{F}$, related to the formation and growth of microcracks that occur in cyclic loadings. We note that being a measure of energy accumulated in the microsctructure, fatigue variable $\mathcal{F}$ grows even under monotonic loading. The form of $\hat{F}$ depends on the absolute value of the power related to stress in the virgin material:

$$
\hat{F}=a(1-\varphi)|(\mathcal{C} \mathbf{E}+b \mathbf{D}): \mathbf{D}|,
$$

where the parameter $a$ in this case is chosen to give a linear dependence of the power of stress.

\subsection{Discretization}

We discretize Eq. (2) in space using the linear finite element method (FEM), where the semidiscrete form is obtained through the Galerkin method. For detailed derivation of the spatial discretization in 2D, we refer to Barros de Moraes et al. (2020). We denote $\ddot{\hat{\mathbf{u}}}=\dot{\hat{\mathbf{v}}}$ and write the semidiscrete form for an element $k$ as

$$
\left\{\begin{array}{l}
\mathbf{M}^{k} \ddot{\hat{\mathbf{u}}}^{k}=\mathbf{K}_{u}^{k} \hat{\mathbf{u}}^{k}+\mathbf{K}_{v}^{k} \hat{\mathbf{v}}^{k}+\mathbf{w}_{a}^{k}+\mathbf{M}^{k} \hat{\mathbf{f}}^{k} \\
\mathbf{M}_{\varphi}^{k} \dot{\hat{\boldsymbol{\varphi}}}^{k}=\left(\mathbf{P}_{\varphi}^{k}+\mathbf{K}_{c}^{k}\right) \hat{\boldsymbol{\varphi}}^{k}+\mathbf{w}_{b}^{k}+\mathbf{w}_{c}^{k} \\
\mathbf{M}_{\mathcal{F}}^{k} \dot{\hat{\mathcal{F}}}^{k}=\mathbf{w}_{d}^{k},
\end{array}\right.
$$

where $\mathbf{M}, \mathbf{M}_{\varphi}$, and $\mathbf{M}_{\mathcal{F}}$ are mass matrices related to displacement, damage, and fatigue, respectively. In the equation of motion, $\mathbf{K}_{u}$ is the damage-degraded elasticity stiffness matrix, $\mathbf{K}_{v}$ is the viscous damping matrix, and $\mathbf{w}_{a}$ is related to the gradient of damage. In the damage evolution equation, $\mathbf{P}_{\varphi}$ includes the Laplacian and potential $\mathcal{H}^{\prime}(\varphi)$. The influence of displacement in damage is represented by $\mathbf{K}_{c}$ and $\mathbf{w}_{b}$. The potential $\mathcal{H}_{f}^{\prime}(\varphi)$ affects the $\mathbf{w}_{c}$ operator, and $\mathbf{w}_{d}$ is the operator on the right-hand side of the fatigue evolution equation. We obtain the global form of operator matrices by the standard assembly operation, and we drop the superscript $k$ in the global sense.

We further discretize the operators in time, adopting a staggered semi-implicit time integration scheme, where nonlinear terms are treated explicitly, thus avoiding the use of iterative methods. Let the solution time interval $[0, T]$ be split in discrete time steps $t_{n}$ with time increments of size $\Delta t=t_{n+1}-t_{n}>0, n=0,1, \ldots$ For the global approximation of any field variable $\mathbf{q}$, we denote $\mathbf{q}_{n+1}=\hat{\mathbf{q}}\left(t_{n+1}\right)$.

We first solve the damage evolution equation and obtain $\boldsymbol{\varphi}_{n+1}$ using the backward Euler scheme, where we treat $\lambda$, displacement, and fatigue explicitly, using values from time step $t_{n}$. Evolution of damage is then obtained by solving the linear system

$$
\left[\mathbf{M}_{\varphi}-\Delta t\left(\mathbf{P}_{\varphi}+\mathbf{K}_{c}\right)\right] \boldsymbol{\varphi}_{n+1}=\mathbf{M}_{\varphi} \boldsymbol{\varphi}_{n}+\Delta t\left(\mathbf{w}_{b}+\mathbf{w}_{c}\right) .
$$

After we update the damage field, we use the Newmark method to solve displacement and velocity in the equation of motion. We denote acceleration and velocity at time $t_{n+1}$ by

$$
\begin{aligned}
& \ddot{\mathbf{u}}_{n+1}=\alpha_{1}\left(\mathbf{u}_{n+1}-\mathbf{u}_{n}\right)-\alpha_{2} \dot{\mathbf{u}}_{n}-\alpha_{3} \ddot{\mathbf{u}}_{n}, \\
& \dot{\mathbf{u}}_{n+1}=\alpha_{4}\left(\mathbf{u}_{n+1}-\mathbf{u}_{n}\right)+\alpha_{5} \dot{\mathbf{u}}_{n}+\alpha_{6} \ddot{\mathbf{u}}_{n},
\end{aligned}
$$

with $\alpha_{i}, i=1,2, \ldots, 6$ written in terms of standard Newmark coefficients $\tilde{\gamma}$ and $\tilde{\beta}$ :

$$
\alpha_{1}=\frac{1}{\tilde{\beta} \Delta t^{2}},
$$

Volume 2, Issue 1, 2021 


$$
\begin{aligned}
& \alpha_{2}=\frac{1}{\tilde{\beta} \Delta t}, \\
& \alpha_{3}=\frac{1-2 \tilde{\beta}}{2 \tilde{\beta}}, \\
& \alpha_{4}=\frac{\tilde{\gamma}}{\tilde{\beta} \Delta t}, \\
& \alpha_{5}=1-\frac{\tilde{\gamma}}{\tilde{\beta}}
\end{aligned}
$$

and

$$
\alpha_{6}=\left(1-\frac{\tilde{\gamma}}{2 \tilde{\beta}}\right) \Delta t
$$

The discrete form of the equation of motion for computing displacements becomes

$$
\begin{aligned}
{\left[\alpha_{1} \mathbf{M}-\mathbf{K}_{u}-\alpha_{4} \mathbf{K}_{v}\right] \mathbf{u}_{n+1} } & =\mathbf{M}\left[\alpha_{3} \ddot{\mathbf{u}}_{n}+\alpha_{2} \dot{\mathbf{u}}_{n}+\alpha_{1} \mathbf{u}_{n}\right] \\
& +\mathbf{K}_{v}\left[\alpha_{6} \ddot{\mathbf{u}}_{n}+\alpha_{5} \dot{\mathbf{u}}_{n}-\alpha_{4} \mathbf{u}_{n}\right]+\mathbf{w}_{a}+\mathbf{M} \mathbf{f}_{n+1} .
\end{aligned}
$$

Then, we update the acceleration and velocity fields using Eqs. (9) and (10), respectively. When imposing prescribed displacement $\overline{\mathbf{u}}\left(t_{n+1}\right)$, we also prescribe corresponding velocity and acceleration at the boundaries using

$$
\overline{\mathbf{u}}_{n+1}=\frac{d^{2}}{d t^{2}} \overline{\mathbf{u}}\left(t_{n+1}\right)
$$

and

$$
\overline{\dot{\mathbf{u}}}_{n+1}=\frac{d}{d t} \overline{\mathbf{u}}\left(t_{n+1}\right),
$$

where the bar symbol represents the prescribed degrees of freedom.

Finally, we use the trapezoidal method to update the fatigue field, obtaining

$$
\mathcal{F}_{n+1}=\mathcal{F}_{n}+\frac{\Delta t}{2} \mathbf{M}_{\mathcal{F}}^{-1}\left[\mathbf{w}_{d}\left(\mathbf{u}_{n+1}, \mathbf{v}_{n+1}, \boldsymbol{\varphi}_{n+1}\right)+\mathbf{w}_{d}\left(\mathbf{u}_{n}, \mathbf{v}_{n}, \boldsymbol{\varphi}_{n}\right)\right] .
$$

We consider the tensile test specimen without notch depicted in Fig. 1. We discretize it with a finite element mesh consisting of 3912 nodes and 7236 linear triangle elements, with smallest element size of $0.614 \mathrm{~mm}$. We constrain one end and apply a prescribed displacement of $4.5 \times 10^{-4} \mathrm{~m} / \mathrm{s}$, with time increments of $\Delta t=5 \times 10^{-4} \mathrm{~s}$, at the other end. We study a material with the Young modulus $E=160 \mathrm{GPa}$, Poisson coefficient $v=0.3$, and density $\rho=7800 \mathrm{~kg} / \mathrm{m}^{3}$, under plane stress conditions with thickness of $h=5 \mathrm{~mm}$. The rate of change of fatigue $a$ is $5 \times 10^{-7} \mathrm{~m}^{2}$, and viscous damping $b$ is $1 \times 10^{8} \mathrm{Ns} / \mathrm{m}^{2}$. The remaining parameters $\gamma$ (phase-field layer width), $g_{c}$ (Griffith energy), and $c$ (rate of change of damage), are chosen in order to construct a set of different representative cases. We focus on those parameters to build the cases because they are the most sensitive and give more uncertainty in damage evolution (Barros de Moraes et al., 2020).

\section{DATA PROCESSING}

In this section, we highlight how to obtain time-series data from phase-field simulations to train and test the learning algorithms. Further, we explore different possibilities of label definitions in the context of failure prediction based on the simulation results. 


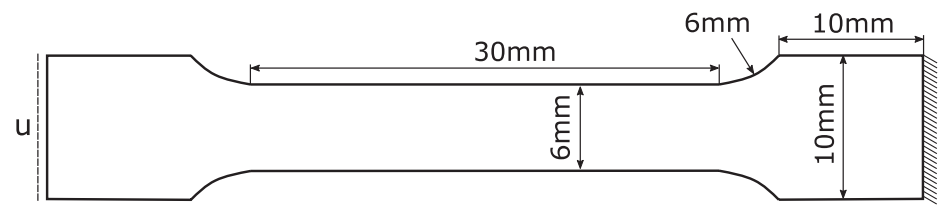

(a)

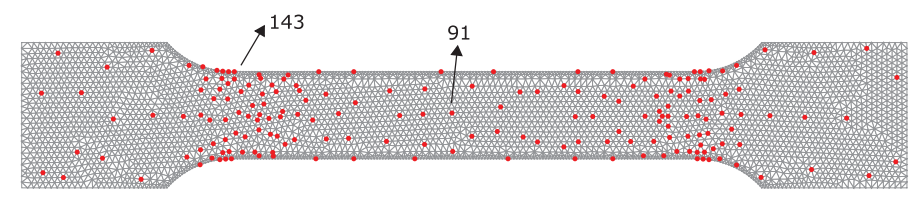

(b)

FIG. 1: Description of geometry and boundary conditions for the tensile test specimen, along with finite element mesh and sensor layout for time-series generation. We highlight two sensor nodes that show different time-series behaviors. (a) Geometry and boundary conditions and (b) mesh and virtual sensor nodes.

\subsection{Time-Series Data Generation}

To generate time-series data, virtual sensing nodes are considered at different locations of the specimen, as shown in Fig. 1. The sensor layout in our tests is simply chosen to provide a coarseto-fine (variable) resolution for the ML framework to calibrate/train and classify time-series data.

Table 1 presents the parameters used to construct each representative failure case or type, for which we plot the damage phase field at failure time in Fig. 2. We also observe three different failure types (i.e., around the fillets, at the middle of the specimen, and in an intermediate region between those), where the effect of changing parameters is clearly noticeable. We observe the different damage evolution in the highlighted sensor nodes shown in Fig. 1 from their time-series data. We plot time-series data from sensor nodes 91 (at the middle of the specimen) and 143 (at one of the fillets), for cases 1,2, and 3, where we observe the different evolution profiles based on each failure type (see Fig. 3).

From the damage phase-field time-series data $\varphi$ at sensing nodes, we then form a feature vector of a pattern as the degradation function $g(\varphi)=(1-\varphi)^{2}$. Thus, patterns are generated using $g(\varphi)$, extracted at sensing nodes for a given time step. Accordingly, a label is assigned to identified patterns at each time step.

Damage $\varphi$ is a proper measure of material failure. However, by defining the feature vector based on the degradation function $g(\varphi)$ instead, we directly measure the material softening, since

TABLE 1: Parameters used in the representative cases

\begin{tabular}{|c|c|c|c|}
\hline Case & $\boldsymbol{\gamma}(\mathbf{m})$ & $\boldsymbol{g}_{\boldsymbol{c}}(\mathbf{N} / \mathbf{m})$ & $\boldsymbol{c}(\mathbf{m} / \mathbf{N s})$ \\
\hline 1 & $3.00 \times 10^{-4}$ & 2700 & $2.00 \times 10^{-6}$ \\
\hline 2 & $2.00 \times 10^{-3}$ & 2700 & $2.00 \times 10^{-6}$ \\
\hline 3 & $5.00 \times 10^{-4}$ & 5400 & $2.00 \times 10^{-6}$ \\
\hline 4 & $5.00 \times 10^{-4}$ & 10800 & $2.00 \times 10^{-6}$ \\
\hline 5 & $2.00 \times 10^{-3}$ & 5400 & $1.00 \times 10^{-6}$ \\
\hline 6 & $2.50 \times 10^{-4}$ & 5400 & $1.00 \times 10^{-6}$ \\
\hline
\end{tabular}

Volume 2, Issue 1, 2021 


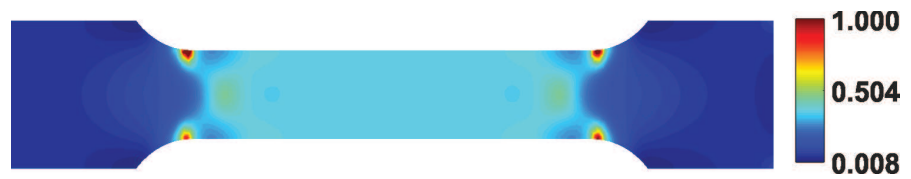

(a)

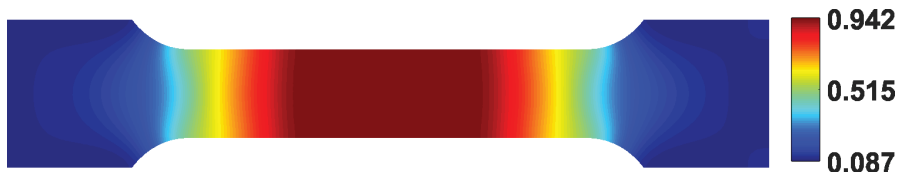

(b)

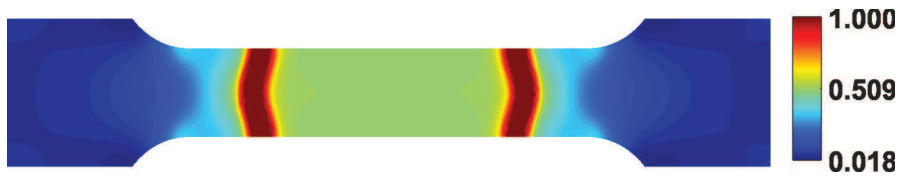

(c)

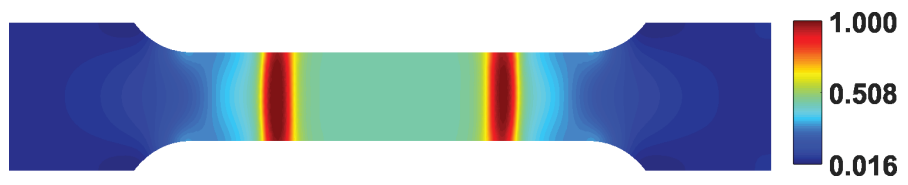

(d)

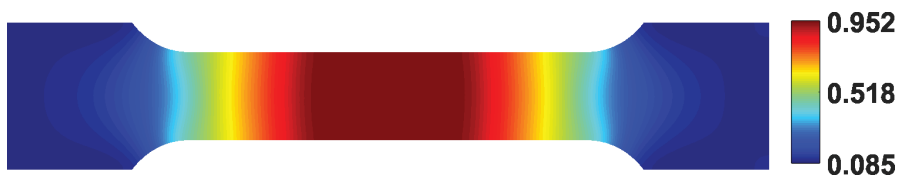

(e)

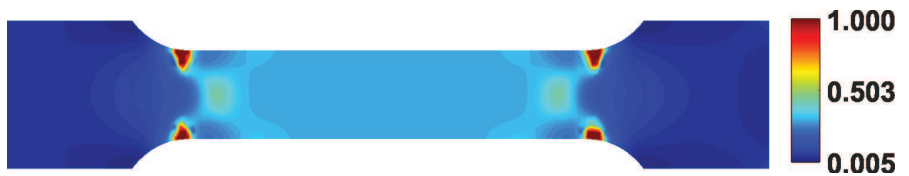

(f)

FIG. 2: Damage phase field for each representative failure case. By changing the parameters $\gamma, g_{c}$, and $c$, we observe different failure types (distinct crack positions and paths), as well as varying dynamics. (a) Case $1, t=0.48 \mathrm{~s}$; (b) case $1, t=0.48 \mathrm{~s}$; (c) case $1, t=0.62 \mathrm{~s}$; (d) case $1, t=0.78 \mathrm{~s}$; (e) case $1, t=0.62 \mathrm{~s}$; (f) case $1, t=0.64 \mathrm{~s}$.

$g(\varphi)$ is the field variable that degrades the constitutive model, thus reducing the component's load-bearing capabilities.

\subsection{Label Definitions}

In the domain of ML, the label is defined as the output of the classification algorithm. We outline different criteria used to generate the labels for the supervised ML algorithms. In the context of failure analysis, labels should reflect the material's capacity to withstand loads, so a first rational 


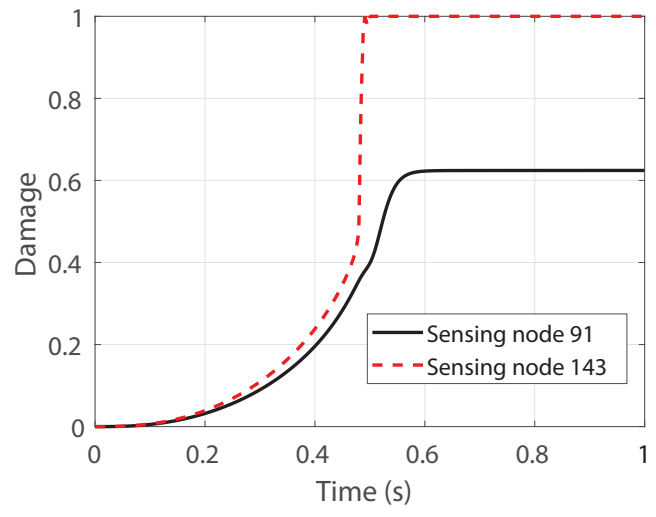

(a)

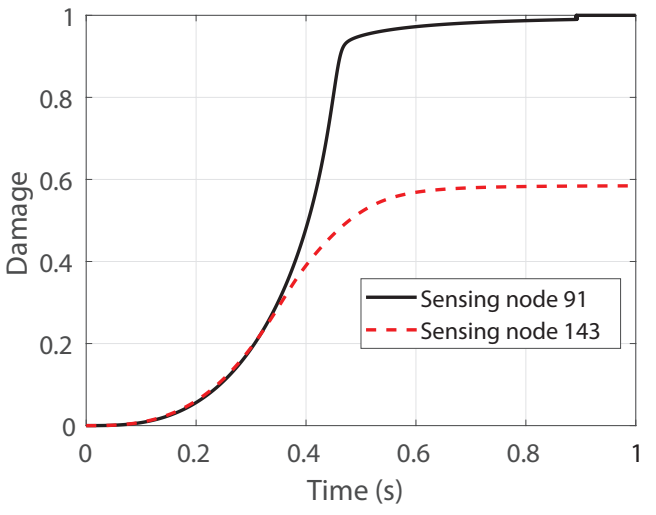

(b)

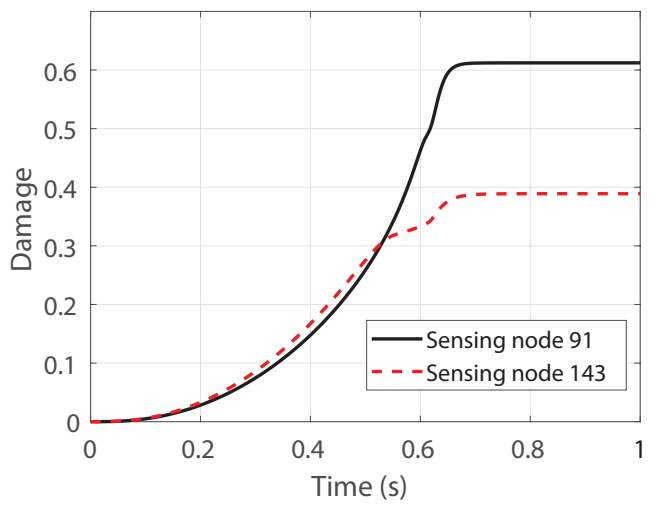

(c)

FIG. 3: Damage phase field time-series data for three cases showing the different evolution of $\varphi$ depending on the virtual sensor node position. (a) Case 1, (b) case 2, (c) case 3.

choice is to define labels based on the load-displacement curve. Besides, we generate labels based on a damage threshold concept with degradation function $g(\varphi)$. Based on noted criteria, each pattern is given a label corresponding to one of multiple classes, namely, no failure (class 1), onset of failure (class 2), and failure (class 3).

\subsubsection{Label Definition According to Load-Displacement Curve}

We start by defining the labels in a binary fashion in order to observe the damage phase field corresponding to a specific label transition. At each time step, a pattern is assigned 0 if there is no failure and 1 if the specimen has fractured, such that we have a label vector

$$
L=\left\{\begin{array}{lllllllll}
0 & 0 & \ldots & 0 & 1 & \ldots & 1 & 1
\end{array}\right\}^{T} .
$$

We assign the labels based on load-displacement curve of the tensile test:

- Label Type 1: Labels are generated based on the maximum force at the load-displacement curve, which may induce to a failure criterion too soon.

Volume 2, Issue 1, 2021 
- Label Type 2: Labels are generated according to the minimum derivative $d f / d u$, which could detect damage too late.

- Label Type 3: Labels are generated based on $85 \%, 90 \%$, and $95 \%$ of maximum force at the load-displacement curve, which yields an intermediate behavior compared to label types 1 and 2.

Figure 4 illustrates the different points, where failure is defined according to the label types, with corresponding damage phase fields. Label types 1 and 2 are too extreme and lead to early

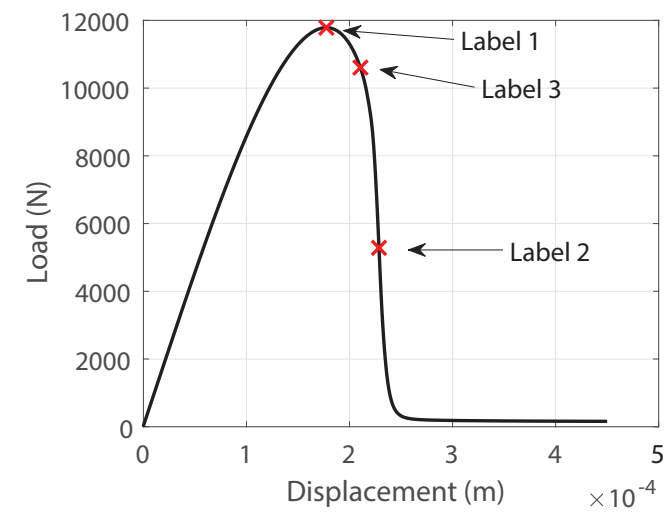

(a)

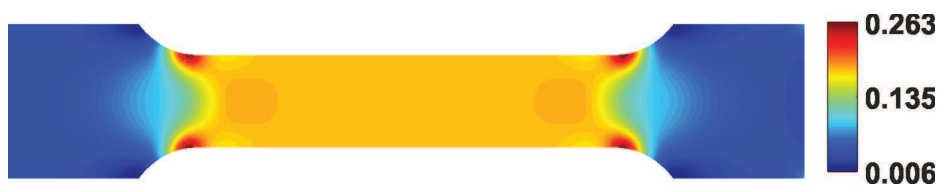

(b)

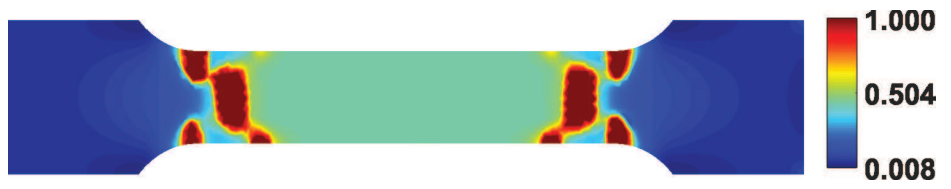

(c)

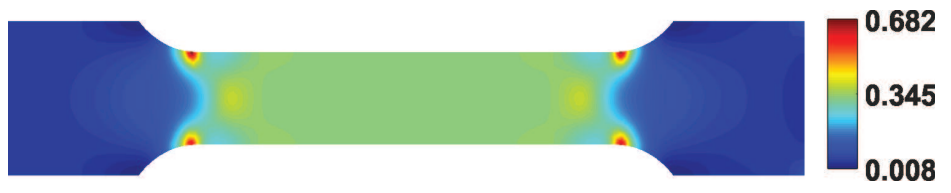

(d)

FIG. 4: (a) Load-displacement curve for case 1, where we identify the three points where the labels change from 0 to 1 according to different criteria. (b)-(d) Respective damage phase fields corresponding to the positions indicated in the curve. We note that label type 3, based on a threshold of $90 \%$ of maximum force, lies between the first two criteria. In label type 1, the damage field is still too smooth, while in label type 2, failure is far too advanced. (a) Load-displacement curve; (b) label 1, $t=0.39 \mathrm{~s}$; (c) label 2, $t=0.51 \mathrm{~s}$; (d) label $3, t=0.47 \mathrm{~s}$. 
and late prediction of failure, respectively. To address this issue, we further improve binary label type 3 by including an intermediate state, the onset of failure, based on percentages of maximum load. Label type 3 then becomes a multiple-label definition, stated here as:

- Multiple Label Type 3: given the labels created based on 90\% and 95\% of maximum force at the load-displacement curve $\left(l_{90}\right.$ and $l_{95}$ ), a pattern $x_{i}$ is assigned to class 1 (no failure) if label of the pattern based on $l_{90}$ and $l_{95}$ is zero, class 2 (onset of failure) when label of the pattern is zero based on $l_{90}$ and one based on $l_{95}$, and class 3 if label of the pattern based on $l_{90}$ and $l_{95}$ is one.

\subsubsection{Label Definition According to Damage Threshold Concept}

We also propose a label definition based on a damage threshold of degradation function $g(\varphi)$, where three different thresholds (i.e., $R_{1}=1, R_{2}=0.92$, and $R_{3}=0.85$ ) are empirically selected based on the simulations. Accordingly, we generate labels by tracking $g(\varphi)$ on all sensing nodes and following the rule:

- Multiple Label Type 4: a given sensor node $S_{i}$ is shown with index $a$ when $R_{1} \geq g(\varphi)>$ $R_{2}$, index $b$ if $R_{2} \geq g(\varphi)>R_{3}$, and index $c$ if $R_{3} \geq g(\varphi)$. Once the noted indices for all sensor nodes at a given time step (i.e., features of pattern $x_{i}$ ) are determined, sum of each index $a$ to $c$ is computed. Pattern $x_{i}$ is then classified as class 1 if summation of index $a$ is larger than that of indices $b$ and $c$, class 2 when summation of index $b$ is greater than summation of indices $a$ and $c$, etc. This label definition is motivated by the neighboring effect concept (i.e., group of sensor nodes), allowing us to eliminate the effect of uncertainty and faulty sensor.

\section{ML ALGORITHMIC FRAMEWORK}

We develop a supervised ML algorithmic framework for interpretation of time-series data generated from the phase-field model. The proposed ML framework presented in Fig. 5 is based on the integration of a PR scheme and ML algorithms. According to the PR scheme, sensor node responses (i.e., time-series data of degradation function $g(\varphi)=(1-\varphi)^{2}$ ) at each time step are represented as a pattern along with the corresponding label. The input to the learning framework is thus a matrix $M$ with dimension $m \times n$, where $m$ denotes the number of time steps, and $n$ represents the number of sensor nodes. Consequently, each row of the matrix denotes a pattern, where the dimension of the PR problem is $n$ (i.e., a pattern with $n$ features).

After generating the noted matrix $M, \mathrm{ML}$ algorithms $k$-NN and ANN are used for failure/damage classification with $m$ identified patterns. By introducing ML algorithms for pattern recognition, as opposed to relying exclusively on the load-displacement curve traditionally employed in failure mechanics, we allow the framework to be generalized, allowing failure detection in complex loading conditions not restricted to the canonical examples presented, and in cases where load-displacement data is not even present. For the same reason, we underline the importance of multiple label type 4, which exclusively depends on damage sensor data.

It should be noted that $k$-NN and ANN are used in this research due to their effective and reliable performance, and these algorithms are computationally efficient. ANN is especially advantageous in this application, where we have a phase-field damage model that is nonlinear, and associated with salient sources of parametric and model-form uncertainties that could be broadcast to the solution. Therefore, we use ANN over other comparable methods to permit a

Volume 2, Issue 1, 2021 


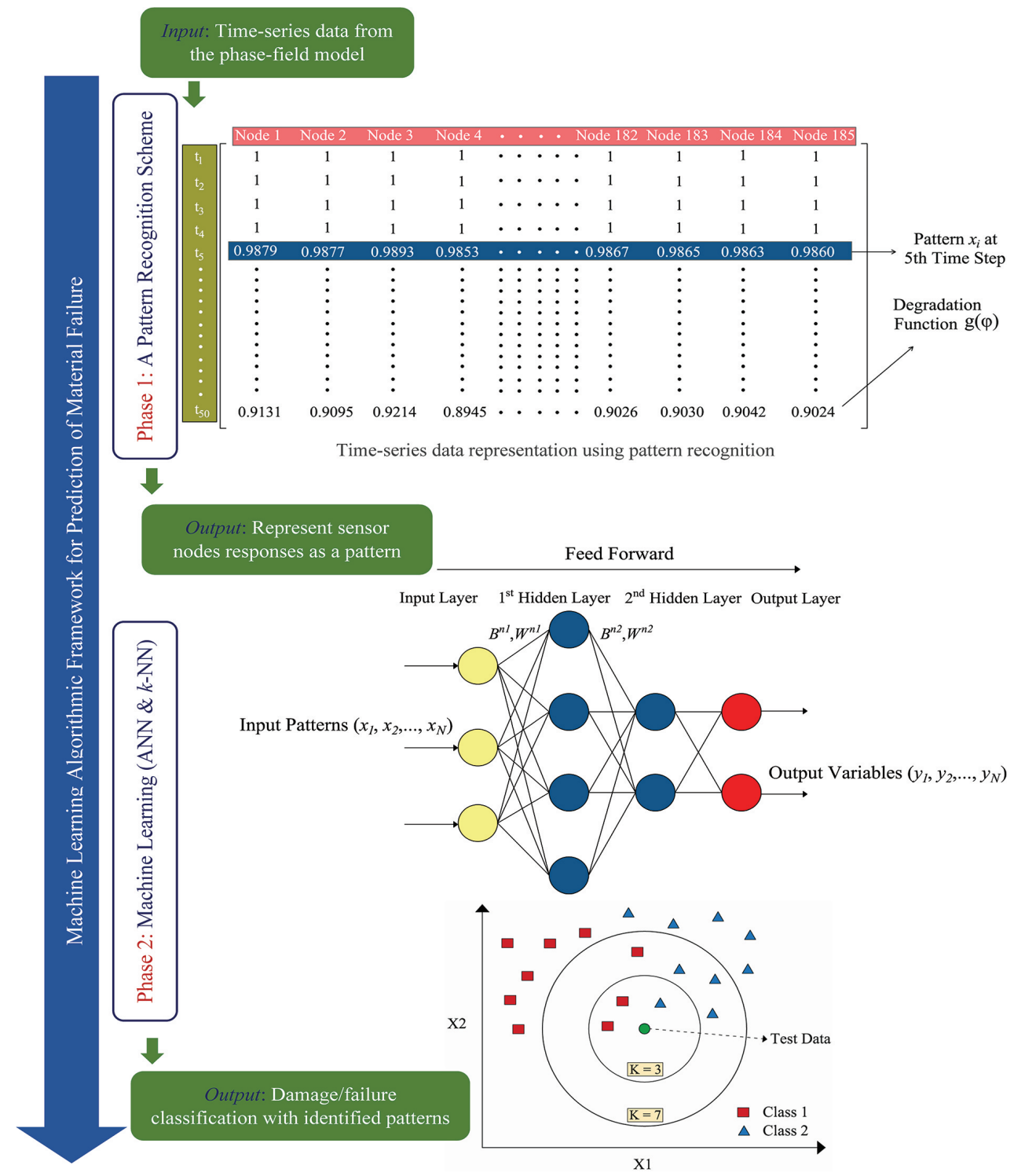

FIG. 5: Schematic illustration of the proposed ML framework. A pattern recognition scheme is introduced to represent time-series data of damage degradation function $g(\varphi)=(1-\varphi)^{2}$ extracted at sensing nodes as a pattern. The $k$-NN and ANN algorithms are employed for failure classification using recognized patterns. In $k$-NN analysis the classification is performed by determining the $k$-nearest vote vector. An ANN provides a map between the inputs and outputs through determination of the weights using input and output patterns.

systematic study of uncertainty propagation and sensitivity of failure detection under noisy data in future works. The theoretical and mathematical details of $k$-NN and ANN can be found in the published literature (Hassoun, 1995; Keller et al., 1985; Weinberger and Saul, 2009; Zhang et al., 1998). 
The dataset for the $k$-NN and ANN analysis is divided into three subsets, namely, training, validation, and test. The training set is used to fit the ML classifiers, while the validation set is used to compute the optimal learning parameters. Performance of the ML classifiers with optimal parameters is then assessed on the test set. The performance of $k$-NN and ANN algorithms is measured using the detection performance rate defined in the following equation:

$$
\text { Classification accuracy }=\frac{\text { Number of patterns correctly classified }}{\text { Total number of identified patterns }} .
$$

Different sizes of data subsets are considered herein to evaluate the effect of such a factor on the performance of the ML algorithms. Accordingly, five different combinations listed below are defined, where the accuracy of $k-\mathrm{NN}$ and ANN is determined based on each combination.

- Comb 1: training \& validation 65\%, and test $35 \%$.

- Comb 2: training \& validation $70 \%$, and test $30 \%$.

- Comb 3: training \& validation $75 \%$, and test $25 \%$.

- Comb 4: training \& validation $80 \%$, and test $20 \%$.

- Comb 5: training \& validation $85 \%$, and test $15 \%$.

\section{RESULTS AND DISCUSSION}

The performance of the developed ML framework in terms of predicting the presence and location/pattern of failure is evaluated with time-series data of degradation function $g(\varphi)=(1-\varphi)^{2}$ generated from the phase-field model. To this aim, the framework is initially trained and tested using each one of six representative failure cases (see Section 3.1), where the presence of failure is detected for each case along with corresponding accuracy. In the next analysis phase, to detect the location of failure, ML algorithms (i.e., $k$-NN and ANN) are trained using data from all six failure cases, and the classification accuracy is determined on test data, leading to identification of the pattern of failure. The following subsections present the classification results of the ML framework, employing multiple labels generated according to multiple label types 3 and 4 (see Section 3.2).

\subsection{Results with $k$-NN}

\subsubsection{Detection of the Presence of Failure}

As noted in Section 4, the proposed ML framework is trained and tested using different sizes of data subsets. For the $k$-NN analysis, $k$-fold cross validation with $k=10$ is used. Patterns identified with the PR scheme, along with corresponding labels, are used as input to the algorithmic framework in order to predict the presence of failure. To further explore the performance of the $k$-NN algorithm, the optimal number of $k$ needs to be determined. In this context, cases 1-3 representing different failure types/locations (see Fig. 2) are considered, based on which the performance of the algorithm is evaluated with varying $k$. The $k$-NN classification results based on multiple label types 3 and 4 are shown in Fig. 6 . As can be seen, by increasing the number of neighbors $(k)$, accuracy decreases. We choose $k=2$ for subsequent analyses, and we will

Volume 2, Issue 1, 2021 


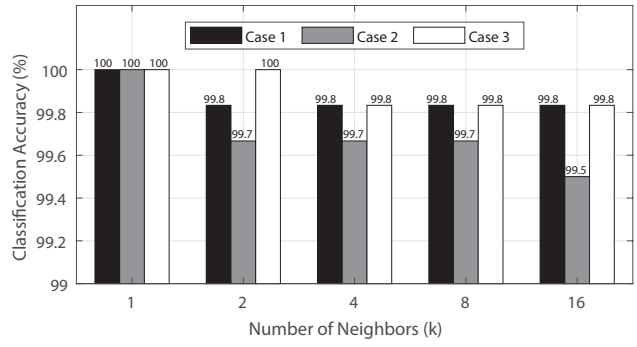

(a)

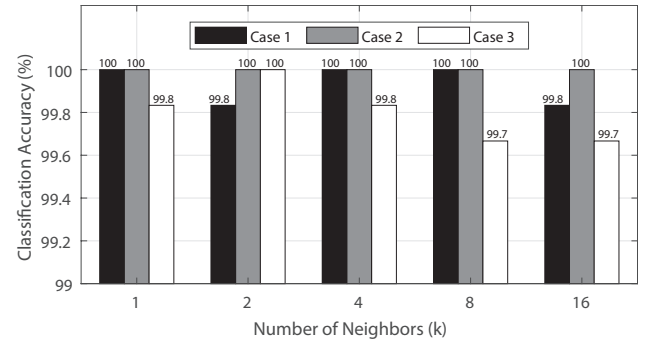

(b)

FIG. 6: $k$-NN classification accuracy with different number of $k$ : (a) accuracy based on multiple label type 3 , (b) accuracy based on multiple label type 4

later check that this is the optimal number of neighbors for detection of failure location. Furthermore, the optimal distance is found to be "cosine," which results in better accuracy compared to other distance functions. Classification results with different sizes of data subsets are presented in Fig. 7 for failure case 3, from which it can be observed that the highest accuracy is achieved based on combinations 2,3 , and 6 , so we choose Comb 2, i.e., training \& validation $70 \%$, and test $30 \%$ for all further results.

The performance of the proposed ML framework employing $k$-NN with multiple labels is assessed for all failure cases (i.e., cases 1 to 6), where the classification accuracy on test data is reported for each case (see Fig. 8). Clearly, the performance of the framework is acceptable such that the highest accuracy based on multiple label types 3 and 4 is $100 \%$ for failure cases 5 and 3 , respectively. To better visualize the classification results, a confusion matrix containing

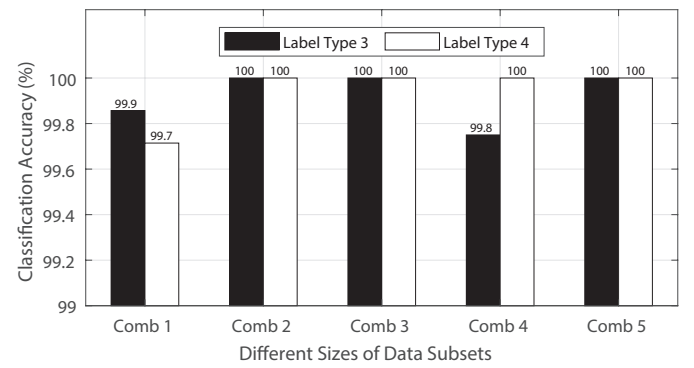

FIG. 7: $k$-NN classification results for failure case 3 with different sizes of data subsets and multiple label types 3 and 4

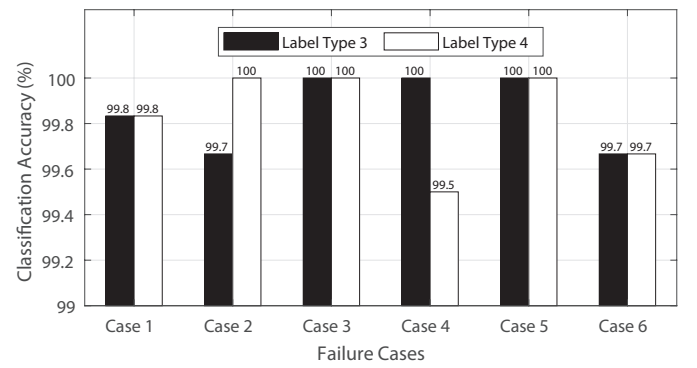

FIG. 8: $k$-NN classification results for different failure cases based on label types 3 and 4 
information about actual and predicted classification is determined. Each column of the confusion matrix represents the patterns in a predicted class, whereas each row denotes the patterns in an actual class. The confusion matrix containing detailed classification results for cases 5 and 1 are depicted in Fig. 9. As can be seen, the $k$-NN method performs well on all classes, including class 2 denoting onset of failure, which is of primary interest for early detection of failure in real-world applications.

\subsubsection{Detection of the Location/Pattern of Failure}

An attempt is made to detect the location of failure, enabling the framework to predict the pattern of failure. In regard to this, three different failure cases, cases 1-3, representing different failure types are considered for the analysis. Accordingly, nine classes/labels are defined, as shown in Table 2, using multiple label type 4 (multiple labels based on damage threshold concept). We also study the effect of different numbers of neighbors $k$ in this context. We present the accuracy

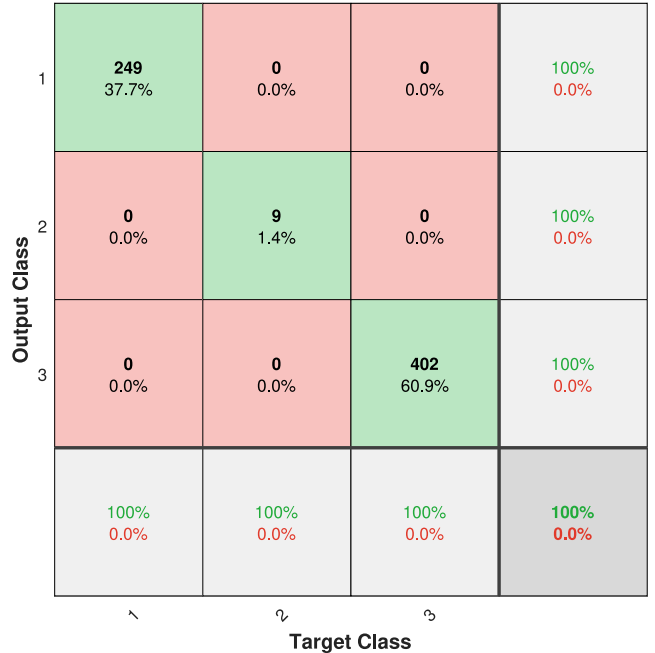

(a)

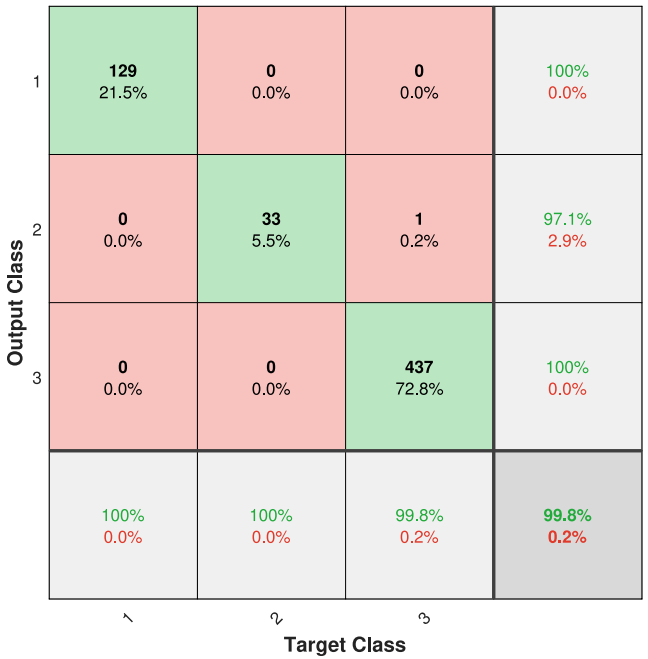

(b)

FIG. 9: Confusion matrix on test data with $k$-NN: (a) case 5 and multiple label type 3, (b) case 1 and multiple label type 4

TABLE 2: Illustration of label/class definition for detection of location/pattern of failure

\begin{tabular}{|c|c|c|c|}
\hline Label/class & Failure case & Label based on label type 4 & New label/class \\
\hline & 1 & 0 & 1 \\
& 1 & 0.5 & 2 \\
Label of a pattern & 1 & 1 & 3 \\
at a given & 2 & 0 & 4 \\
time step & 2 & 0.5 & 5 \\
& 2 & 1 & 6 \\
& 3 & 0 & 7 \\
& 3 & 0.5 & 9 \\
\hline
\end{tabular}

Volume 2, Issue 1, 2021 
results in Fig. 10, in which we observe that $k=2$ is indeed the optimal number of neighbors, corroborating the choice made in the previous section.

The confusion matrix showing the classification results is presented in Fig. 11. As can be observed, the total accuracy is reported as $98.3 \%$ using multiple labels/classes shown in Table 2 ( $k$-NN detects the location of failure with $98.3 \%$ accuracy). Results indicate the overall efficient

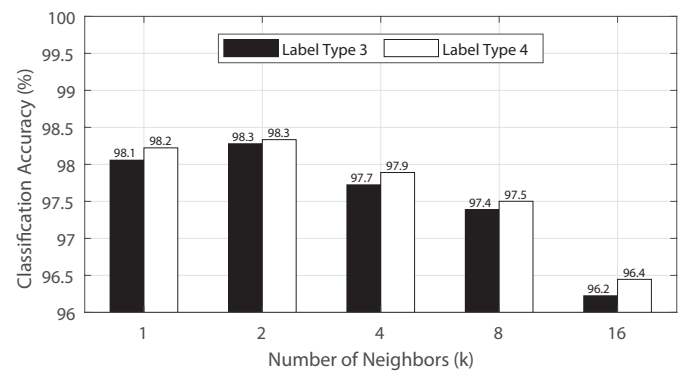

FIG. 10: $k$-NN classification accuracy with different numbers of $k$ based on multiple label types 3 and 4

\begin{tabular}{|c|c|c|c|c|c|c|c|c|c|c|}
\hline \multicolumn{11}{|c|}{ Confusion Matrix } \\
\hline 1 & $\begin{array}{c}117 \\
6.5 \%\end{array}$ & $\begin{array}{c}0 \\
0.0 \%\end{array}$ & $\begin{array}{c}0 \\
0.0 \%\end{array}$ & $\begin{array}{c}3 \\
0.2 \%\end{array}$ & $\begin{array}{c}0 \\
0.0 \%\end{array}$ & $\begin{array}{c}0 \\
0.0 \%\end{array}$ & $\begin{array}{c}12 \\
0.7 \%\end{array}$ & $\begin{array}{c}0 \\
0.0 \%\end{array}$ & $\begin{array}{c}0 \\
0.0 \%\end{array}$ & $\begin{array}{l}88.6 \% \\
11.4 \%\end{array}$ \\
\hline 2 & $\begin{array}{c}0 \\
0.0 \%\end{array}$ & $\begin{array}{c}32 \\
1.8 \%\end{array}$ & $\begin{array}{c}1 \\
0.1 \%\end{array}$ & $\begin{array}{c}0 \\
0.0 \%\end{array}$ & $\begin{array}{c}0 \\
0.0 \%\end{array}$ & $\begin{array}{c}0 \\
0.0 \%\end{array}$ & $\begin{array}{c}0 \\
0.0 \%\end{array}$ & $\begin{array}{c}0 \\
0.0 \%\end{array}$ & $\begin{array}{c}0 \\
0.0 \%\end{array}$ & $\begin{array}{c}97.0 \% \\
3.0 \%\end{array}$ \\
\hline 3 & $\begin{array}{c}0 \\
0.0 \%\end{array}$ & $\begin{array}{c}0 \\
0.0 \%\end{array}$ & $\begin{array}{c}430 \\
23.9 \%\end{array}$ & $\begin{array}{c}0 \\
0.0 \%\end{array}$ & $\begin{array}{c}0 \\
0.0 \%\end{array}$ & $\begin{array}{c}0 \\
0.0 \%\end{array}$ & $\begin{array}{c}0 \\
0.0 \%\end{array}$ & $\begin{array}{c}0 \\
0.0 \%\end{array}$ & $\begin{array}{c}0 \\
0.0 \%\end{array}$ & $\begin{array}{l}100 \% \\
0.0 \%\end{array}$ \\
\hline 4 & $\begin{array}{c}4 \\
0.2 \%\end{array}$ & $\begin{array}{c}0 \\
0.0 \%\end{array}$ & $\begin{array}{c}0 \\
0.0 \%\end{array}$ & $\begin{array}{c}107 \\
5.9 \%\end{array}$ & $\begin{array}{c}0 \\
0.0 \%\end{array}$ & $\begin{array}{c}0 \\
0.0 \%\end{array}$ & $\begin{array}{c}6 \\
0.3 \%\end{array}$ & $\begin{array}{c}0 \\
0.0 \%\end{array}$ & $\begin{array}{c}0 \\
0.0 \%\end{array}$ & $\begin{array}{c}91.5 \% \\
8.5 \%\end{array}$ \\
\hline & $\begin{array}{c}0 \\
0.0 \%\end{array}$ & $\begin{array}{c}0 \\
0.0 \%\end{array}$ & $\begin{array}{c}0 \\
0.0 \%\end{array}$ & $\begin{array}{c}0 \\
0.0 \%\end{array}$ & $\begin{array}{c}25 \\
1.4 \%\end{array}$ & $\begin{array}{c}0 \\
0.0 \%\end{array}$ & $\begin{array}{c}0 \\
0.0 \%\end{array}$ & $\begin{array}{c}0 \\
0.0 \%\end{array}$ & $\begin{array}{c}0 \\
0.0 \%\end{array}$ & $\begin{array}{l}100 \% \\
0.0 \%\end{array}$ \\
\hline & $\begin{array}{c}0 \\
0.0 \%\end{array}$ & $\begin{array}{c}0 \\
0.0 \%\end{array}$ & $\begin{array}{c}0 \\
0.0 \%\end{array}$ & $\begin{array}{c}0 \\
0.0 \%\end{array}$ & $\begin{array}{c}1 \\
0.1 \%\end{array}$ & $\begin{array}{c}495 \\
27.5 \%\end{array}$ & $\begin{array}{c}0 \\
0.0 \%\end{array}$ & $\begin{array}{c}0 \\
0.0 \%\end{array}$ & $\begin{array}{c}0 \\
0.0 \%\end{array}$ & $\begin{array}{c}99.8 \% \\
0.2 \%\end{array}$ \\
\hline 7 & $\begin{array}{c}2 \\
0.1 \%\end{array}$ & $\begin{array}{c}0 \\
0.0 \%\end{array}$ & $\begin{array}{c}0 \\
0.0 \%\end{array}$ & $\begin{array}{c}1 \\
0.1 \%\end{array}$ & $\begin{array}{c}0 \\
0.0 \%\end{array}$ & $\begin{array}{c}0 \\
0.0 \%\end{array}$ & $\begin{array}{c}115 \\
6.4 \%\end{array}$ & $\begin{array}{c}0 \\
0.0 \%\end{array}$ & $\begin{array}{c}0 \\
0.0 \%\end{array}$ & $\begin{array}{c}97.5 \% \\
2.5 \%\end{array}$ \\
\hline 8 & $\begin{array}{c}0 \\
0.0 \%\end{array}$ & $\begin{array}{c}0 \\
0.0 \%\end{array}$ & $\begin{array}{c}0 \\
0.0 \%\end{array}$ & $\begin{array}{c}0 \\
0.0 \%\end{array}$ & $\begin{array}{c}0 \\
0.0 \%\end{array}$ & $\begin{array}{c}0 \\
0.0 \%\end{array}$ & $\begin{array}{c}0 \\
0.0 \%\end{array}$ & $\begin{array}{c}47 \\
2.6 \%\end{array}$ & $\begin{array}{c}0 \\
0.0 \%\end{array}$ & $\begin{array}{l}100 \% \\
0.0 \%\end{array}$ \\
\hline 9 & $\begin{array}{c}0 \\
0.0 \%\end{array}$ & $\begin{array}{c}0 \\
0.0 \%\end{array}$ & $\begin{array}{c}0 \\
0.0 \%\end{array}$ & $\begin{array}{c}0 \\
0.0 \%\end{array}$ & $\begin{array}{c}0 \\
0.0 \%\end{array}$ & $\begin{array}{c}0 \\
0.0 \%\end{array}$ & $\begin{array}{c}0 \\
0.0 \%\end{array}$ & $\begin{array}{c}0 \\
0.0 \%\end{array}$ & $\begin{array}{c}403 \\
22.4 \%\end{array}$ & $\begin{array}{l}100 \% \\
0.0 \%\end{array}$ \\
\hline & $\begin{array}{c}95.1 \% \\
4.9 \%\end{array}$ & $\begin{array}{l}100 \% \\
0.0 \%\end{array}$ & $\begin{array}{c}99.8 \% \\
0.2 \%\end{array}$ & $\begin{array}{c}96.4 \% \\
3.6 \%\end{array}$ & $\begin{array}{c}96.2 \% \\
3.8 \%\end{array}$ & $\begin{array}{c}100 \% \\
0.0 \%\end{array}$ & $\begin{array}{l}86.5 \% \\
13.5 \%\end{array}$ & $\begin{array}{l}100 \% \\
0.0 \%\end{array}$ & $\begin{array}{c}100 \% \\
0.0 \%\end{array}$ & $\begin{array}{c}98.3 \% \\
1.7 \%\end{array}$ \\
\hline & 1 & 2 & 3 & $\star$ & 5 & 6 & 1 & 8 & 9 & \\
\hline & & & & & ara & lass & & & & \\
\hline
\end{tabular}

FIG. 11: Confusion matrix with $k$-NN classification results for detection of location/pattern of failure based on multiple label type 4 
performance of $k$-NN to detect the onset of failure (classes 2, 5, and 8) and failure (classes 3,6 , and 9). The incorrect classifications are more concentrated in classes denoting no failure (classes 1, 4, and 7), where the method incorrectly identifies the location of failure in a few data points, because in early stages of the simulation the damage field is similar among the different cases. This is not an issue since the critical part is the onset of failure. Moreover, the lowest classification accuracy is $86.5 \%$ for class 7 (see Fig. 11), which is an acceptable performance for a classification algorithm.

\subsection{Results with ANN}

\subsubsection{Detection of the Presence of Failure}

The performance of the proposed ML framework employing ANN algorithm is evaluated in terms of detecting the presence of failure. On this basis, a two-layer (i.e., one hidden layer with five neurons and output layer) feed-forward neural network with Sigmoid classifier/activation function is used. Classification results based on multiple label types 3 and 4 are presented in Fig. 12, from which it can be seen that ANN leads to comparable accuracy compared to $k$-NN (see Fig. 8). Detailed classification results with ANN are presented in a confusion matrix shown in Fig. 13. Results indicate that ANN effectively detects the presence of failure with a total accuracy of $99.90 \%$ and $100 \%$ using multiple label types 3 and 4, respectively.

\subsubsection{Detection of the Location/Pattern of Failure}

Once the presence of failure is detected, ANN algorithm is employed to identify the location/pattern of failure. On this basis, multiple labels defined in Table 2 are used for supervised classification with ANN algorithm. A two-layer (i.e., one hidden layer with five neurons and output layer) feed-forward neural network with Sigmoid activation function is used as the ANN architecture to detect the location of failure. The confusion matrix with detailed classification results is presented in Fig. 14, from which it can be observed that the location/pattern of failure can be successfully detected using ANN with high accuracy. Similarly to $k$-NN, the majority of misclassifications in ANN belong to classes representing no failure (classes 1 and 4 in Fig. 14), due to the similarity of the damage field prior to damage localization and crack initiation. In the other classes, ANN still performs successfully, with a minimum accuracy of $79.3 \%$ when detecting the onset of failure (class 5).

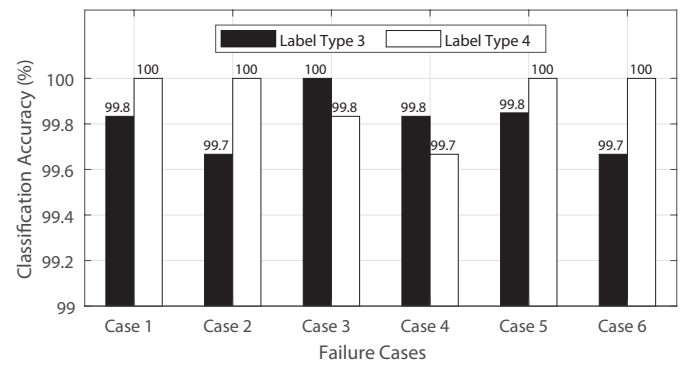

FIG. 12: ANN classification results for different failure cases based on multiple label types 3 and 4

Volume 2, Issue 1, 2021 


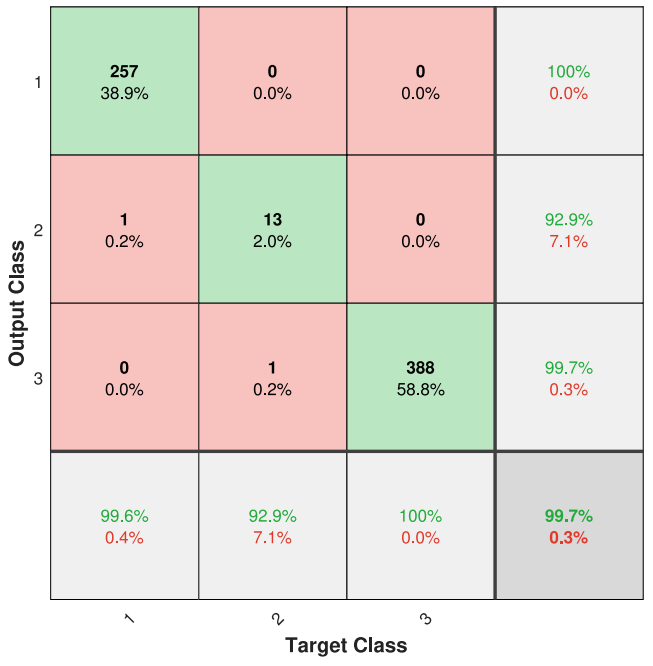

(a)

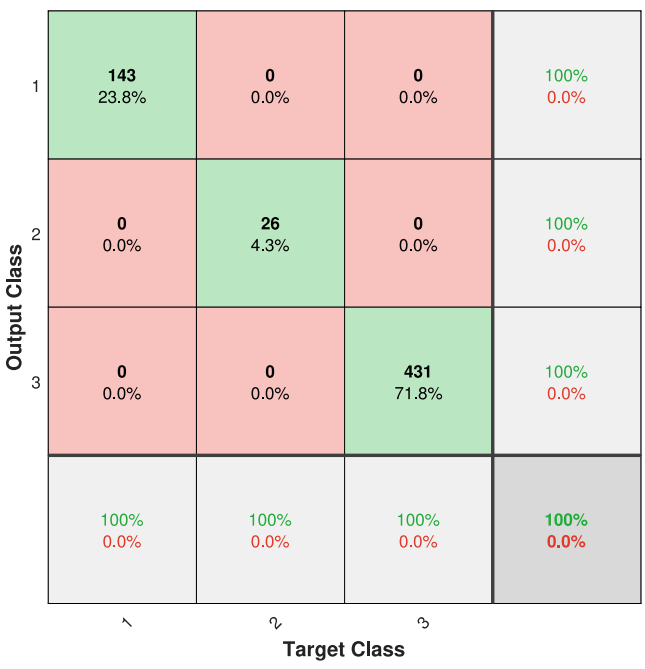

(b)

FIG. 13: Confusion matrix on test data with ANN: (a) case 5 and multiple label type 3, (b) case 1 and multiple label type 4

\subsection{Discussion of Deterministic Results}

From the observation of classification results employing $k$-NN and ANN to predict failure in damage phase-field models, we see consistent results with high accuracy. This is especially the case of $k$-NN, which shows less uncertainty in classifying the patterns. Yet, we must highlight that the nature of data used in this work plays a major role in such satisfactory results. Several nuances and complications of classification algorithms, such as variance-bias tradeoff, sensitivity, and distribution/number of data points, are either justified, or less critical, due to characteristics of phase field models.

In essence, phase-field models are smooth representations of sharp, discontinuous interfaces. In the case of damage modeling, cracks are smoothened in the phase field as an intrinsic approximation of the model. Therefore, as opposed to real data measurements, phase-field solutions evaluated at virtual sensing nodes are naturally smooth, introduce low noise, and consequently lead to less variance in the predictions.

In this regard, the choice of number and position of virtual sensing nodes was performed by an a priori study of failure patterns from Fig. 2. Different configurations of training data led to little change of classification results. The distribution of sensors considered expert opinions to provide the framework with data points associated with different modes of failure. Such assessment considers that the placement of sensors in regions around the bases of the specimen will not contribute to accuracy since there is no damage accumulation in such areas. We remark that for a specific failure case, with model parameters appropriately inferred from experiments, fewer sensor nodes would be required, placed preferably in critical areas (e.g., around the corners and in the central region).

With respect to the specific algorithms used in this work, the nature of phase-field data aids in the tradeoff between bias and variance. Both $k$-NN and ANN have been extensively studied in the literature, and the bias-variance behavior is well understood. For $k$-NN, it has been demonstrated 


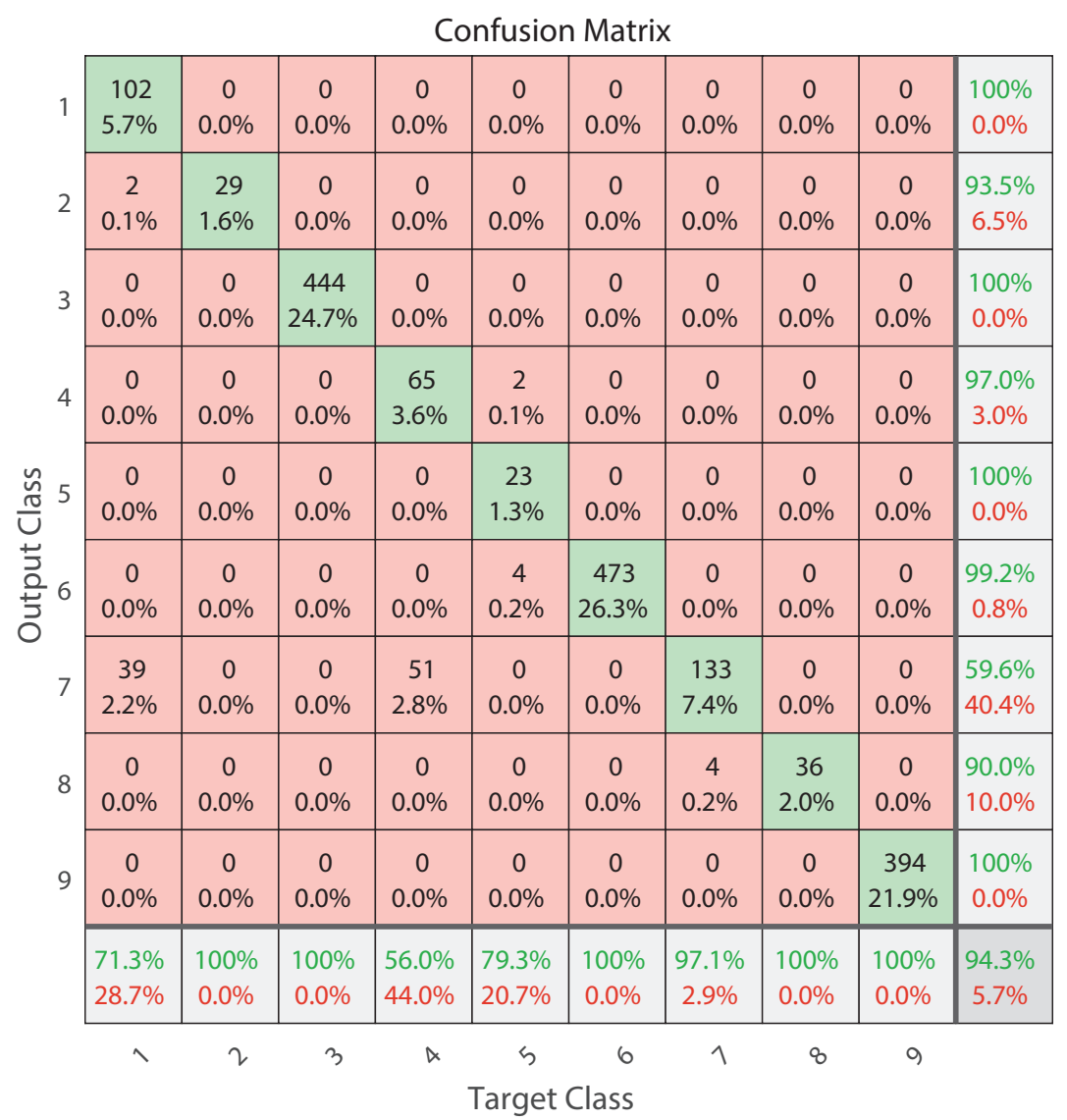

FIG. 14: Confusion matrix with ANN classification results for detection of location/pattern of failure based on multiple label Type 4

analytically that bias increases, and variance decreases with an increasing number of neighbors (Friedman et al., 2001; Geman et al., 1992). Since the smoothness of phase-field solutions does not introduce substantial variance, the choice of $k=2$ makes the tradeoff by reducing the bias. In the case of ANN, we reduce bias by increasing the number of hidden units (Geman et al., 1992), so the number of neurons used is also justified.

Another source of potential errors is related to the number of time steps and their distribution among the classes. This issue shows specifically when predicting onset of failure, the most critical part of the failure process. Classification errors in this case may be due to fewer data points available, since the onset of failure occurs rapidly. A possible remedy would be to refine the time-integration steps around the onset of failure. Additionally, we have shown that the algorithm incorrectly classifies cases of no failure between different fracture patterns due to enhanced smoothness early on the simulation. However, this issue is less catastrophic when compared to erroneously detecting the onset of failure, regardless of the fracture pattern.

Overall, the framework worked effectively with deterministic data, due to the nature of phase-field models, and reasonable choice of $k$-NN and ANN parameters to compromise the relationship between bias and variance. Since phase field models present smooth features with

Volume 2, Issue 1, 2021 
low variance, we further assess the robustness of the framework by adding noise to the damage field solutions.

\subsection{Uncertainty Quantification}

The results presented in previous sections consisted of smooth, deterministic input data in a single run of the ML algorithms. In this section we propagate the uncertainty associated to data sampling and randomness related to the algorithms, and we assess the robustness and accuracy of both methods to the variability in data through the addition of Gaussian noise. This approach aims to verify the effectiveness of the framework to handle real-world data.

\subsubsection{Algorithmic Randomness}

We first study the propagation of uncertainties related to the algorithms, still using deterministic data. Such randomness appears in both $k$-NN and ANN due to the random division of timeseries data into training, validation, and test sets. We need to choose random division to avoid bias, specially in damage data that shows pronounced temporal evolution trends. Furthermore, ANN also presents another source of uncertainty related to the random initialization of weights and biases in each neuron.

We use the Monte Carlo (MC) method to run multiple classification problems for each algorithm and to compute the expected total classification accuracy and standard deviation. We use multiple label type 3 and run 1000 simulations for detection of failure presence in each case (cases 1-6), and run an additional 1000 classifications for the detection of failure location, using classes from Table 2 . We show the results in Table 3 . We observe that $k$-NN performs better than ANN in this setting since ANN incorporates another level of uncertainty from the random guesses of neuron parameters. The randomness of data division does not affect the performance of either method in the failure location problem. For failure location, ANN is less accurate but still within acceptable range.

\subsubsection{Noisy Data}

To assess the performance of the proposed ML framework with noisy data, time-series data are corrupted by adding Gaussian distributed noise with different standard deviations to damage $\varphi$ data. We run $1000 \mathrm{MC}$ simulations for $k$-NN and ANN algorithms using multiple label type 3

TABLE 3: Total classification accuracy mean and standard deviation from algorithmic randomness (\%)

\begin{tabular}{|c|c|c|c|c|}
\hline & \multicolumn{2}{|c|}{$k$-NN } & \multicolumn{2}{c|}{ ANN } \\
\cline { 2 - 5 } & Mean & Std. Dev & Mean & Std. Dev \\
\hline Failure presence—case 1 & 99.86 & 0.16 & 99.82 & 1.53 \\
\hline Failure presence—case 2 & 99.86 & 0.16 & 99.88 & 0.14 \\
\hline Failure presence—case 3 & 99.87 & 0.14 & 99.84 & 0.27 \\
\hline Failure presence-case 4 & 99.86 & 0.16 & 99.82 & 0.31 \\
\hline Failure presence-case 5 & 99.87 & 0.14 & 99.89 & 0.15 \\
\hline Failure presence-case 6 & 99.86 & 0.15 & 99.88 & 0.16 \\
\hline Failure location-cases 1/2/3 & 98.28 & 0.31 & 84.58 & 6.00 \\
\hline
\end{tabular}


and compute the total accuracy expectation and standard deviation. We propagate uncertainty for failure presence detection in case 3 , and for detection of failure location, and show the results in Fig. 15.

We observe that increasing noise levels decreases the mean accuracy while enlarging the uncertainty range for both methods. We see that even low-intensity noise drops the accuracy to lower levels when compared to mean values based only on algorithmic randomness. In other words, input data dominates over algorithms' uncertainty. For failure presence detection in case 3 , we see that ANN performs better than $k$-NN for low noise levels, while $k$-NN is more robust under higher noise magnitudes, resulting in higher mean accuracy and lower standard deviation. For case 3, the lowest accuracy was still above $75 \%$, showing robustness with three classes. Similarly, when we look at detection of failure location, ANN is superior to $k$-NN for low noise levels and shows higher mean accuracy even for high noise intensity, yet with more uncertainty. However, we cannot claim good performance of the algorithms with nine classes under high noise levels under this specific choice of algorithmic setup. This motivates a more systematic approach to uncertainty and sensitivity of ML algorithms under noisy phase-field data, and it shall be the focus of future studies.

\section{SUMMARY AND CONCLUSIONS}

This paper presents a phase field-based machine learning (ML) framework developed to predict failure of brittle materials. Time-series data are generated according to nodal damage results from finite element simulations of a tensile test specimen. We assessed the performance of the proposed ML framework employing PR scheme and ML algorithms ( $k$-NN and ANN) for different failure types and with multiple labels generated based on load-displacement curve and damage threshold concept. We draw the following conclusions from the study:

- Results indicate the acceptable performance of the proposed framework with multiple labels, in which a PR scheme is effectively used to represent time-series data of degradation function $g(\varphi)=(1-\varphi)^{2}$ as a pattern. This choice of time-series data is effective since it directly complies with the material softening behavior.

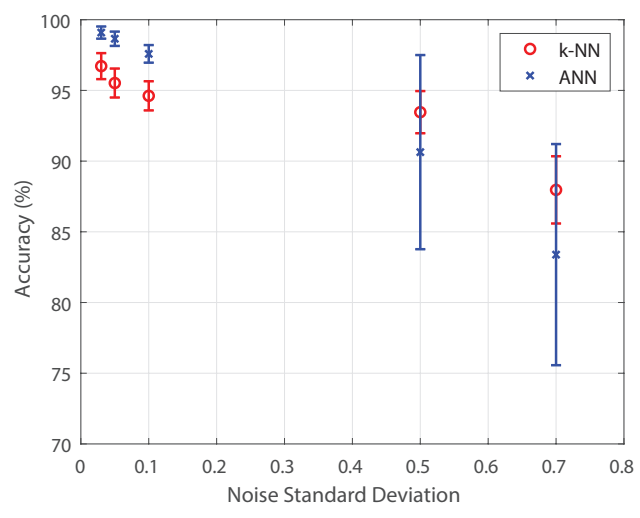

(a)

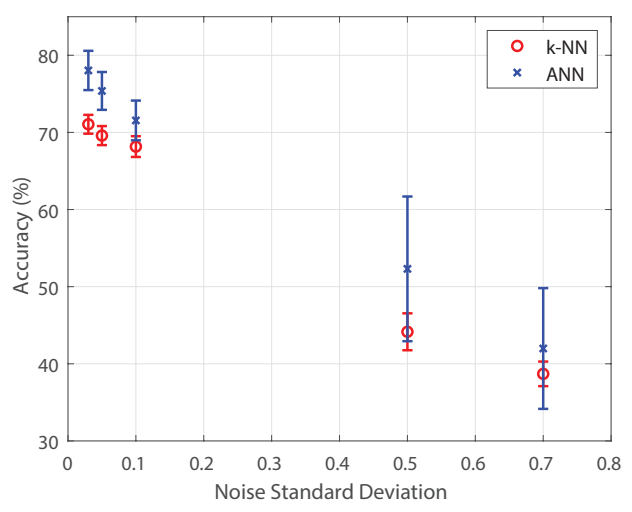

(b)

FIG. 15: Mean total classification accuracy and standard deviation for (a) detection of failure presence, case 3 , and, (b) detection of failure location

Volume 2, Issue 1, 2021 
- Both $k$-NN and ANN were efficient in predicting the presence and location of failure. The majority of errors in detection of failure location were concentrated in classes representing no failure due to smoothness and similarity of damage field early in the simulations.

- Uncertainty related to input data noise dominates over algorithmic randomness uncertainty. The framework showed robustness to noise when detecting failure presence and showed acceptable accuracy with low noise levels when predicting failure location. In general, with noisy data ANN outperforms $k$-NN.

Results of this study demonstrate the satisfactory performance of the developed algorithmic framework and the applicability of ML for failure prediction with damage phase-field time-series data. This study aims to promote the state-of-the-art in data-driven failure prediction indicating the practical application of ML for failure detection in brittle materials. Findings from this study are expected to advance the development of data-driven frameworks capable of establishing a direct relationship between the classification accuracy and damage phase-field parameters. In other words, in such frameworks, the output of ML framework can be further used as input to the damage phase-field model to identify the parameters leading to failure/damage. This will significantly result in enhancing the data-driven failure prediction framework's performance.

It is acknowledged that the results presented in this paper are based on time-series data extracted at virtual sensing nodes across the test specimen. Yet, the proposed ML framework can be effectively applied to a system of real (i.e., nonvirtual) sensing nodes. It is expected that the developed algorithmic framework will perform satisfactorily with real sensor data.

It is worth pointing out that although the mathematical approach presented in this research is deterministic, there is an uncertainty associated with the proposed ML framework because of the random nature of such intelligent algorithms. In addition, the time-series data used in this study are smooth. Nonetheless, this issue cannot always be valid since near failure/damage, regions time-series data can become noisy due to stress/strain concentration in such regions. Future works will focus on addressing the noted issues. Besides, the focus of future studies will be on the evaluation of the developed framework with the inclusion of plasticity/visco-elastoplasticity (Suzuki and Muñoz-Rojas, 2014; Suzuki et al., 2016; Varghaei et al., 2019) in the damage and fatigue phase-field model, combined with efficient and stable long time integration schemes (Zhou et al., 2019).

\section{ACKNOWLEDGMENTS}

This work was supported by the ARO Young Investigator Program Award (W911NF-19-1-0444), the MURI/ARO Award (W911NF-15-1-0562), the National Science Foundation Award (DMS1923201), and the AFOSR Young Investigator Program Award (FA9550-17-1-0150).

\section{REFERENCES}

Al Azzawi, D., Moncayo, H., Perhinschi, M.G., Perez, A., and Togayev, A., Comparison of ImmunityBased Schemes for Aircraft Failure Detection and Identification, Eng. Appl. Artif. Intel., vol. 52, pp. 181193, 2016.

Allen, S.M. and Cahn, J.W., A Microscopic Theory for Antiphase Boundary Motion and Its Application to Antiphase Domain Coarsening, Acta Metal., vol. 27, no. 6, pp. 1085-1095, 1979.

Ambati, M., Gerasimov, T., and De Lorenzis, L., Phase-Field Modeling of Ductile Fracture, Comput. Mech., vol. 55, no. 5, pp. 1017-1040, 2015. 
Ambati, M., Kruse, R., and De Lorenzis, L., A Phase-Field Model for Ductile Fracture at Finite Strains and Its Experimental Verification, Comput. Mech., vol. 57, no. 1, pp. 149-167, 2016.

Amendola, G., Fabrizio, M., and Golden, J.M., Thermomechanics of Damage and Fatigue by a Phase Field Model, J. Therm. Stress., vol. 39, no. 5, pp. 487-499, 2016.

Barros de Moraes, E.A., Zayernouri, M., and Meerschaert, M.M., An Integrated Sensitivity-Uncertainty Quantification Framework for Stochastic Phase-Field Modeling of Material Damage, Int. J. Numer. Methods Eng., 2020. (in press)

Boldrini, J., Barros de Moraes, E., Chiarelli, L., Fumes, F., and Bittencourt, M., A Non-Isothermal Thermodynamically Consistent Phase Field Framework for Structural Damage and Fatigue, Comput. Methods Appl. Mech. Eng., vol. 312, pp. 395-427, 2016.

Borden, M.J., Hughes, T.J., Landis, C.M., and Verhoosel, C.V., A Higher-Order Phase-Field Model for Brittle Fracture: Formulation and Analysis within the Isogeometric Analysis Framework, Comput. Methods Appl. Mech. Eng., vol. 273, pp. 100-118, 2014.

Borden, M.J., Verhoosel, C.V., Scott, M.A., Hughes, T.J., and Landis, C.M., A Phase-Field Description of Dynamic Brittle Fracture, Comput. Methods Appl. Mech. Eng., vols. 217-220, pp. 77-95, 2012.

Cahn, J.W. and Hilliard, J.E., Free Energy of a Nonuniform System. I. Interfacial Free Energy, J. Chem. Phys., vol. 28, no. 2, pp. 258-267, 1958.

Caputo, M. and Fabrizio, M., Damage and Fatigue Described by a Fractional Derivative Model, J. Comput. Phys., vol. 293, pp. 400-408, 2015.

Carrara, P., Ambati, M., Alessi, R., and De Lorenzis, L., A Framework to Model the Fatigue Behavior of Brittle Materials Based on a Variational Phase-Field Approach, Comput. Methods Appl. Mech. Eng., p. 112731, 2019.

Chiarelli, L., Fumes, F., Barros de Moraes, E., Haveroth, G., Boldrini, J.L., and Bittencourt, M.L., Comparison of High Order Finite Element and Discontinuous Galerkin Methods for Phase Field Equations: Application to Structural Damage, Comput. Math. Appl., vol. 74, no. 7, pp. 1542-1564, 2017.

Friedman, J., Hastie, T., and Tibshirani, R., The Elements of Statistical Learning, vol. 1, New York, NY: Springer, 2001.

Geman, S., Bienenstock, E., and Doursat, R., Neural Networks and the Bias/Variance Dilemma, Neural Comput., vol. 4, no. 1, pp. 1-58, 1992.

Griffith, A.A., VI. The Phenomena of Rupture and Flow in Solids, Philos. Transact. Royal Soc. A, vol. 221, nos. 582-593, pp. 163-198, 1921.

Hassoun, M.H., Fundamentals of Artificial Neural Networks, Cambridge, MA: MIT Press, 1995.

Haveroth, G.A., Barros de Moraes, E.A., Boldrini, J.L., and Bittencourt, M.L., Comparison of Semi and Fully-Implicit Time Integration Schemes Applied to a Damage and Fatigue Phase Field Model, Lat. Am. J. Solids Struct., vol. 15, no. 5, 2018.

Hofacker, M. and Miehe, C., A Phase Field Model of Dynamic Fracture: Robust Field Updates for the Analysis of Complex Crack Patterns: A Phase Field Model of Dynamic Fracture, Int. J. Numer. Methods Eng., vol. 93, no. 3, pp. 276-301, 2013.

Hunter, A., Moore, B.A., Mudunuru, M., Chau, V., Tchoua, R., Nyshadham, C., Karra, S., O’Malley, D., Rougier, E., Viswanathan, H., and Srinivasan, G., Reduced-Order Modeling through Machine Learning and Graph-Theoretic Approaches for Brittle Fracture Applications, Comput. Mater. Sci., vol. 157, pp. 87-98, 2019.

Keller, J.M., Gray, M.R., and Givens, J.A., A Fuzzy K-Nearest Neighbor Algorithm, IEEE Transact. Sys., Man, Cybernet., no. 4, pp. 580-585, 1985.

Lemaitre, J. and Desmorat, R., Engineering Damage Mechanics: Ductile, Creep, Fatigue and Brittle Failures, New York, NY: Springer Science \& Business Media, 2005.

Volume 2, Issue 1, 2021 
Lim, H.J. and Sohn, H., Online Fatigue Crack Quantification and Prognosis Using Nonlinear Ultrasonic Modulation and Artificial Neural Network, Sensors and Smart Structures Technologies for Civil, Mechanical, and Aerospace Systems 2018, vol. 10598, Denver, CO, March 5-8, 2018.

Lim, H.J., Sohn, H., and Kim, Y., Data-Driven Fatigue Crack Quantification and Prognosis Using Nonlinear Ultrasonic Modulation, Mech. Sys. Signal Proc., vol. 109, pp. 185-195, 2018.

Lima, E.A.B.F., Oden, J.T., and Almeida, R.C., A Hybrid Ten-Species Phase-Field Model of Tumor Growth, Math. Models Methods Appl. Sci., vol. 24, no. 13, pp. 2569-2599, 2014.

Liu, Y.Y., Ju, Y.F., Duan, C.D., and Zhao, X.F., Structure Damage Diagnosis Using Neural Network and Feature Fusion, Eng. Appl. Artif. Intel., vol. 24, no. 1, pp. 87-92, 2011.

Miehe, C., Hofacker, M., and Welschinger, F., A Phase Field Model for Rate-Independent Crack Propagation: Robust Algorithmic Implementation Based on Operator Splits, Comput. Methods Appl. Mech. Eng., vol. 199, nos. 45-48, pp. 2765-2778, 2010a.

Miehe, C., Welschinger, F., and Hofacker, M., Thermodynamically Consistent Phase-Field Models of Fracture: Variational Principles and Multi-Field FE Implementations, Int. J. Numer. Methods Eng., vol. 83, no. 10, pp. 1273-1311, 2010b.

Moore, B.A., Rougier, E., O’Malley, D., Srinivasan, G., Hunter, A., and Viswanathan, H., Predictive Modeling of Dynamic Fracture Growth in Brittle Materials with Machine Learning, Comput. Mater. Sci., vol. 148, pp. 46-53, 2018.

Papanikolaou, S., Tzimas, M., Reid, A.C., and Langer, S.A., Spatial Strain Correlations, Machine Learning, and Deformation History in Crystal Plasticity, Phys. Rev. E, vol. 99, no. 5, p. 053003, 2019.

Rovinelli, A., Sangid, M.D., Proudhon, H., and Ludwig, W., Using Machine Learning and a Data-Driven Approach to Identify the Small Fatigue Crack Driving Force in Polycrystalline Materials, NPJ Comput. Mater., vol. 4, no. 1, p. 35, 2018.

Saeidpour, A., Chorzepa, M.G., Christian, J., and Durham, S., Parameterized Fragility Assessment of Bridges Subjected to Hurricane Events Using Metamodels and Multiple Environmental Parameters, J. Infrastruct. Sys., vol. 24, no. 4, p. 04018031, 2018.

Salehi, H. and Burgueño, R., Emerging Artificial Intelligence Methods in Structural Engineering, Eng. Struct., vol. 171, pp. 170-189, 2018.

Salehi, H., Das, S., Chakrabartty, S., Biswas, S., and Burgueño, R., Damage Identification in Aircraft Structures with Self-Powered Sensing Technology: A Machine Learning Approach, Struct. Control Health Monitor, vol. 25, no. 12, p. e2262, 2018a.

Salehi, H., Das, S., Chakrabartty, S., Biswas, S., and Burgueño, R., Structural Damage Identification Using Image-Based Pattern Recognition on Event-Based Binary Data Generated from Self-Powered Sensor Networks, Struct. Control Health Monitor., vol. 25, no. 4, p. e2135, 2018b.

Salehi, H., Biswas, S., and Burgueño, R., Data Interpretation Framework Integrating Machine Learning and Pattern Recognition for Self-Powered Data-Driven Damage Identification with Harvested Energy Variations, Eng. Appl. Artif. Intel., vol. 86, pp. 136-153, 2019a.

Salehi, H., Das, S., Chakrabartty, S., Biswas, S., and Burgueño, R., An Algorithmic Framework for Reconstruction of Time-Delayed and Incomplete Binary Signals from an Energy-Lean Structural Health Monitoring System, Eng. Struct., vol. 180, pp. 603-620, 2019 b.

Santos, A., Figueiredo, E., Silva, M., Sales, C., and Costa, J., Machine Learning Algorithms for Damage Detection: Kernel-Based Approaches, J. Sound Vibrat., vol. 363, pp. 584-599, 2016.

Schwarzer, M., Rogan, B., Ruan, Y., Song, Z., Lee, D.Y., Percus, A.G., Chau, V.T., Moore, B.A., Rougier, E., Viswanathan, H., and Srinivasan, G., Learning to Fail: Predicting Fracture Evolution in Brittle Material Models Using Recurrent Graph Convolutional Neural Networks, Comput. Mater. Sci., vol. 162, pp. 322-332, 2019.

Seiler, M., Linse, T., Hantschke, P., and Kästner, M., An Efficient Phase-Field Model for Fatigue Fracture 
in Ductile Materials, 2019. arXiv: 1903.06465

Shen, Z.H., Wang, J.J., Jiang, J.Y., Huang, S.X., Lin, Y.H., Nan, C.W., Chen, L.Q., and Shen, Y., PhaseField Modeling and Machine Learning of Electric-Thermal-Mechanical Breakdown of Polymer-Based Dielectrics, Nat. Commun., vol. 10, no. 1, p. 1843, 2019.

Silva, M., Santos, A., Figueiredo, E., Santos, R., Sales, C., and Costa, J.C., A Novel Unsupervised Approach Based on a Genetic Algorithm for Structural Damage Detection in Bridges, Eng. Appl. Artif. Intel., vol. 52, pp. 168-180, 2016.

Sun, P., Xu, J., and Zhang, L., Full Eulerian Finite Element Method of a Phase Field Model for Fluid-Structure Interaction Problem, Comput. Fluids, vol. 90, pp. 1-8, 2014.

Suzuki, J. and Muñoz-Rojas, P., Transient Analysis of Geometrically Non-Linear Trusses Considering Coupled Plasticity and Damage, Tenth World Congress on Computational Mechanics, vol. 1, p. 98, Sanya, China, December 5-7, 2014.

Suzuki, J., Zayernouri, M., Bittencourt, M., and Karniadakis, G., Fractional-Order Uniaxial Visco-ElastoPlastic Models for Structural Analysis, Comput. Methods Appl. Mech. Eng., vol. 308, pp. 443-467, 2016.

Teichert, G., Natarajan, A., Van der Ven, A., and Garikipati, K., Machine Learning Materials Physics: Integrable Deep Neural Networks Enable Scale Bridging by Learning Free Energy Functions, Comput. Methods Appl. Mech. Eng., vol. 353, pp. 201-216, 2019.

Varghaei, P., Kharazmi, E., Suzuki, J.L., and Zayernouri, M., Vibration Analysis of Geometrically Nonlinear and Fractional Viscoelastic Cantilever Beams, 2019. arXiv: 1909.02142,

Weinberger, K.Q. and Saul, L.K., Distance Metric Learning for Large Margin Nearest Neighbor Classification, J. Machine Learn. Res., vol. 10, pp. 207-244, 2009.

Wootton, A.J., Butcher, J.B., Kyriacou, T., Day, C.R., and Haycock, P.W., Structural Health Monitoring of a Footbridge Using Echo State Networks and NARMAX, Eng. Appl. Artif. Intel., vol. 64, pp. 152-163, 2017.

Yabansu, Y.C., Steinmetz, P., Hötzer, J., Kalidindi, S.R., and Nestler, B., Extraction of Reduced-Order Process-Structure Linkages from Phase-Field Simulations, Acta Mater., vol. 124, pp. 182-194, 2017.

Yue, P., Feng, J.J., Liu, C., and Shen, J., A Diffuse-Interface Method for Simulating Two-Phase Flows of Complex Fluids, J. Fluid Mech., vol. 515, p. 293-317, 2004.

Zhang, G., Patuwo, B.E., and Hu, M.Y., Forecasting with Artificial Neural Networks: The State of the Art, Int. J. Forecast., vol. 14, no. 1, pp. 35-62, 1998.

Zhou, Y., Suzuki, J.L., Zhang, C., and Zayernouri, M., Fast Imex Time Integration of Nonlinear Stiff Fractional Differential Equations, 2019. arXiv: 1909.04132

Volume 2, Issue 1, 2021 